\title{
Generators of invariant linear system on tropical curves for finite isometry group
}

\author{
Song JuAe
}

\begin{abstract}
For a tropical curve $\Gamma$ and a finite subgroup $K$ of the isometry group of $\Gamma$, we prove, extending the work by Haase, Musiker and $\mathrm{Yu}$ (3]), that the $K$-invariant part of the complete linear system associated to a $K$-invariant effective divisor on $\Gamma$ is finitely generated.
\end{abstract}

\section{Introduction}

Let $R(D)$ denote the set consisting of rational functions corresponding to the complete linear system $|D|$ for an effective divisor $D$ on a tropical curve $\Gamma$, where a tropical curve means a metric graph possibly with unbounded edges. $R(D)$ becomes a tropical semimodule. The projective space $R(D) / \boldsymbol{R}$ is naturally identified with the complete linear system $|D|$. Haase, Musiker and Yu showed that $R(D)$ is finitely generated ([3, Theorem 6]). A tropical subsemimodule $R^{\prime}$ of $R(D)$ corresponds to a linear subspace $\Lambda$ of $|D|$. This linear subspace $\Lambda$ is called a linear system associated to $R^{\prime}$.

In this paper, we recall some basic facts of tropical curves in Section 2. Then in Section 3, we observe the $K$-invariant set $R(D)^{K}$ of $R(D)$ and prove that $R(D)^{K}$ is actually finitely generated, where $K$ is a finite subgroup of the isometry group of $\Gamma$. Our proof is basically analogous to that of [3], but it is not perfectly compatible, i.e. the $K$-invariant set $S^{K}$ of the generator set $S$ of $R(D)$ defined in [3, Lemma 6] is not a generator set of $R(D)^{K}$. We find such a set corresponding to $S$, which we call $S(D)_{K}$. The condition defining $S(D)_{K}$ is tangibly given from geometric information. Also, the construction of a harmonic morphism with degree $|K|$ from $\Gamma$ to the quotient tropical curve $\Gamma^{\prime}$ of $\Gamma$ by $K$ precedes. We follow Chan's natural construction ([2]) with a little bit of adaptation. Finally, using the harmonic morphism, we prove that 
$R(D)^{K}$ is finitely generated as a tropical semimodule in our main theorem (Theorem 3.11). When $D$ is $K$-invariant, we can identify $R(D)^{K} / \boldsymbol{R}$ with the $K$-invariant linear subsystem $|D|^{K}$ and then $|D|^{K}$ is finitely generated by $S(D)_{K} / \boldsymbol{R}$.

\section{Preliminaries}

In this section, we briefly recall the theories of tropical curves ([5]), divisors on tropical curves $([5])$, harmonic morphisms of tropical curves ([2], [3], [4]), and chip-firing moves on tropical curves ([3]), which we need later.

\subsection{Tropical curves}

In this paper, a graph means an unweighted, finite connected nonempty multigraph. Note that we allow the existence of loops. For a graph $G$, the sets of vertices and edges are denoted by $V(G)$ and $E(G)$, respectively. The valence $\operatorname{val}(v)$ of a vertex $v$ of $G$ is the number of edges emanating from $v$, where we count each loop as two. A vertex $v$ of $G$ is a leaf end if $v$ has valence one. A leaf edge is an edge of $G$ having a leaf end.

An edge-weighted graph $(G, l)$ is the pair of a graph $G$ and a function $l: E(G) \rightarrow \boldsymbol{R}_{>0} \cup\{\infty\}$ called a length function, where $l$ can take the value $\infty$ on only leaf edges. A tropical curve is the underlying $\infty$-metric space of an edge-weighted graph $(G, l)$. For a point $x$ on a tropical curve $\Gamma$ obtained from $(G, l)$, if the distances between $x$ and all points on $\Gamma$ other than $x$ are infinity, then $x$ is called a point at infinity, else, $x$ is said to be a finite point. For the above tropical curve $\Gamma,(G, l)$ is said to be its model. There are many possible models for $\Gamma$. We construct a model $\left(G_{\circ}, l_{\circ}\right)$ called the canonical model of $\Gamma$ as follows: when $\Gamma$ is a circle, we determine $V\left(G_{\circ}\right)$ as the set consisting of one arbitrary point on $\Gamma$, else when $\Gamma$ is the $\infty$-metric space obtained from only one edge with length of $\infty, V\left(G_{\circ}\right)$ consists of the two endpoints of $\Gamma$ (those are points at infinity) and an any point on $\Gamma$ as the origin, else, generally, we determine $V\left(G_{\circ}\right):=\{x \in \Gamma \mid \operatorname{val}(x) \neq 2\}$, where the valence $\operatorname{val}(x)$ is the number of connected components of $U_{x} \backslash\{x\}$ with $U_{x}$ being any sufficiently small connected neighborhood of $x$ in $\Gamma$. Since connected components of $\Gamma \backslash V\left(G_{\circ}\right)$ consist of open intervals, whose lengths determine the length function $l_{\circ}$. If a model $(G, l)$ of $\Gamma$ has no loops, then $(G, l)$ is said to be a loopless model of $\Gamma$. For a model $(G, l)$ of $\Gamma$, the loopless model for 
$(G, l)$ is obtained by regarding all midpoints of loops of $G$ as vertices and by adding them to the set of vertices of $G$. The loopless model for the canonical model of a tropical curve is called the canonical loopless model.

For terminology, in a tropical curve $\Gamma$, an edge of $\Gamma$ means an edge of the underlying graph $G_{\circ}$ of the canonical model $\left(G_{\circ}, l_{\circ}\right)$. Let $e$ be an edge of $\Gamma$ which is not a loop. We regard $e$ as a closed subset of $\Gamma$, i.e., including the endpoints $v_{1}, v_{2}$ of $e$. The relative interior of $e$ is $e^{\circ}=e \backslash\left\{v_{1}, v_{2}\right\}$. For a point $x$ on $\Gamma$, a half-edge of $x$ is a connected component of $U_{x} \backslash\{x\}$ with any sufficiently small connected neighborhood $U_{x}$ of $x$.

For a model $(G, l)$ of a tropical curve $\Gamma$, we frequently identify a vertex $v$ (resp. an edge $e$ ) of $G$ with the point corresponding to $v$ on $\Gamma$ (resp. the closed subset corresponding to $e$ of $\Gamma$ ).

\subsection{Divisors on tropical curves}

Let $\Gamma$ be a tropical curve. An element of the free abelian group $\operatorname{Div}(\Gamma)$ generated by points on $\Gamma$ is called a divisor on $\Gamma$. For a divisor $D$ on $\Gamma$, its degree $\operatorname{deg}(D)$ is defined by the sum of the coefficients over all points on $\Gamma$. We write the coefficient at $x$ as $D(x)$. A divisor $D$ on $\Gamma$ is said to be effective if $D(x) \geq 0$ for any $x$ in $\Gamma$. If $D$ is effective, we write simply $D \geq 0$. The set of points on $\Gamma$ where the coefficient(s) of $D$ is not zero is called the support of $D$ and written as $\operatorname{supp}(D)$.

A rational function on $\Gamma$ is a constant function of $-\infty$ or a piecewise linear function with integer slopes and with a finite number of pieces, taking the value $\pm \infty$ only at points at infinity. $\operatorname{Rat}(\Gamma)$ denotes the set of rational functions on $\Gamma$. For a point $x$ on $\Gamma$ and $f$ in $\operatorname{Rat}(\Gamma)$ which is not constant $-\infty$, the sum of the outgoing slopes of $f$ at $x$ is denoted by $\operatorname{ord}_{x}(f)$. If $x$ is a point at infinity and $f$ is infinite there, we define $\operatorname{ord}_{x}(f)$ as the outgoing slope from any sufficiently small connected neighborhood of $x$. Note when $\Gamma$ is a singleton, for any $f$ in $\operatorname{Rat}(\Gamma)$, we define $\operatorname{ord}_{x}(f):=0$. This sum is 0 for all but finite number of points on $\Gamma$, and thus

$$
\operatorname{div}(f):=\sum_{x \in \Gamma} \operatorname{ord}_{x}(f) \cdot x
$$

is a divisor on $\Gamma$, which is called a principal divisor. Two divisors $D$ and $E$ on $\Gamma$ are said to be linearly equivalent if $D-E$ is a principal divisor. We handle the values $\infty$ and $-\infty$ as follows: let $f, g$ in $\operatorname{Rat}(\Gamma)$ take the value $\infty$ and $-\infty$ at a point $x$ at infinity on $\Gamma$ respectively, and $y$ be any 
point in any sufficiently small neighborhood of $x$. When $\operatorname{ord}_{x}(f)+\operatorname{ord}_{x}(g)$ is negative, then $(f \odot g)(x):=\infty$. When $\operatorname{ord}_{x}(f)+\operatorname{ord}_{x}(g)$ is positive, then $(f \odot g)(x):=-\infty$. Remark that the constant function of $-\infty$ on $\Gamma$ dose not determine a principal divisor. For a divisor $D$ on $\Gamma$, the complete linear system $|D|$ is defined by the set of effective divisors on $\Gamma$ being linearly equivalent to $D$.

The set of $\boldsymbol{R}$ with two tropical operations:

$$
a \oplus b:=\max \{a, b\} \quad \text { and } \quad a \odot b:=a+b
$$

becomes a semiring called the tropical semiring, where both $a$ and $b$ are in $\boldsymbol{R}$. For a divisor $D$ on a tropical curve, let $R(D)$ be the set of rational functions $f \neq-\infty$ such that $D+\operatorname{div}(f)$ is effective. When $\operatorname{deg}(D)$ is negative, $|D|$ is empty, so is $R(D)$. Otherwise, from the argument in Section 3 of [3], $D$ is not empty and consequently so is $R(D)$. Hereafter, we treat only divisors of nonnegative degree.

Lemma 2.2.1 (cf. [3, Lemma 4]). $R(D)$ becomes a tropical semimodule on $\boldsymbol{R}$ by extending above tropical operations onto functions, giving pointwise sum and product.

By the definition of $\operatorname{ord}_{x}(f)$ for a point $x$ at infinity and $f$ in $\operatorname{Rat}(\Gamma)$, we can prove Lemma 2.2.1 in the same way of [3, Lemma 4].

For a tropical subsemimodule $M$ of $(\boldsymbol{R} \cup\{ \pm \infty\})^{\Gamma}$ (or of $\boldsymbol{R}^{\Gamma}$ ), $f$ in $M$ is called an extremal of $M$ when it implies $f=g_{1}$ or $f=g_{2}$ that any $g_{1}$ and $g_{2}$ in $M$ satisfies $f=g_{1} \oplus g_{2}$.

Remark 2.2.2 ([3, Proposition 8]). Any finitely generated tropical subsemimodule $\widetilde{M}$ of $\boldsymbol{R}^{\Gamma}$ is generated by the extremals of $\widetilde{M}$.

With the adaptation for $\pm \infty$, we can prove the following lemma in same way as the above remark.

Lemma 2.2.3. Any finitely generated tropical subsemimodule $M$ of $R(D) \subset$ $(\boldsymbol{R} \cup\{ \pm \infty\})^{\Gamma}$ is generated by the extremals of $M$.

\subsection{Harmonic morphisms}

Let $\Gamma, \Gamma^{\prime}$ be tropical curves, respectively, and $\varphi: \Gamma \rightarrow \Gamma^{\prime}$ be a continuous map. The map $\varphi$ is called a morphism if there exist a model $(G, l)$ of $\Gamma$ and 
a model $\left(G^{\prime}, l^{\prime}\right)$ of $\Gamma^{\prime}$ such that the image of the set of vertices of $G$ by $\varphi$ is a subset of the set of vertices of $G^{\prime}$, the inverse image of the relative interior of any edge of $G^{\prime}$ by $\varphi$ is the union of the relative interiors of a finite number of edges of $G$ and the restriction of $\varphi$ to any edge $e$ of $G$ is a dilation by some non-negative integer factor $\operatorname{deg}_{e}(\varphi)$. Note that the dilation factor on $e$ with $\operatorname{deg}_{e}(\varphi) \neq 0$ represents the ratio of the distance of the images of any two points $x$ and $y$ except points at infinity on $e$ to that of original $x$ and $y$. If an edge $e$ is mapped to a vertex of $G^{\prime}$ by $\varphi$, then $\operatorname{deg}_{e}(\varphi)=0$. The morphism $\varphi$ is said to be finite if $\operatorname{deg}_{e}(\varphi)>0$ for any edge $e$ of $G$. For any half-edge $h$ of any point on $\Gamma$, we define $\operatorname{deg}_{h}(\varphi)$ as $\operatorname{deg}_{e}(\varphi)$, where $e$ is the edge of $G$ containing $h$.

Let $\Gamma^{\prime}$ be not a singleton and $x$ a point on $\Gamma$. The morphism $\varphi$ is harmonic at $x$ if the number

$$
\operatorname{deg}_{x}(\varphi):=\sum_{h \mapsto h^{\prime}} \operatorname{deg}_{h}(\varphi)
$$

is independent of the choice of half-edge $h^{\prime}$ emanating from $\varphi(x)$, where $h$ is a connected component of the inverse image of $h^{\prime}$ by $\varphi$. The morphism $\varphi$ is harmonic if it is harmonic at all points on $\Gamma$. One can check that if $\varphi$ is a finite harmonic morphism, then the number

$$
\operatorname{deg}(\varphi):=\sum_{x \mapsto x^{\prime}} \operatorname{deg}_{x}(\varphi)
$$

is independent of the choice of a point $x^{\prime}$ on $\Gamma^{\prime}$, and is said the degree of $\varphi$, where $x$ is an element of the inverse image of $x^{\prime}$ by $\varphi$. If $\Gamma^{\prime}$ is a singleton and $\Gamma$ is not a singleton, for any point $x$ on $\Gamma$, we define $\operatorname{deg}_{x}(\varphi)$ as zero so that we regard $\varphi$ as a harmonic morphism of degree zero. If both $\Gamma$ and $\Gamma^{\prime}$ are singletons, we regard $\varphi$ as a harmonic morphism which can have any number of degree.

Let $\varphi: \Gamma \rightarrow \Gamma^{\prime}$ be a harmonic morphism between tropical curves. For $f$ in $\operatorname{Rat}(\Gamma)$, the push-forward of $f$ is the function $\varphi_{*} f: \Gamma^{\prime} \rightarrow \boldsymbol{R} \cup\{ \pm \infty\}$ defined by

$$
\varphi_{*} f\left(x^{\prime}\right):=\sum_{\substack{x \in \Gamma \\ \varphi(x)=x^{\prime}}} \operatorname{deg}_{x}(\varphi) \cdot f(x) .
$$

The pull-back of $f^{\prime}$ in $\operatorname{Rat}\left(\Gamma^{\prime}\right)$ is the function $\varphi^{*} f^{\prime}: \Gamma \rightarrow \boldsymbol{R} \cup\{ \pm \infty\}$ defined by $\varphi_{*} f^{\prime}:=f^{\prime} \circ \varphi$. We define the push-forward on divisors $\varphi_{*}: \operatorname{Div}(\Gamma) \rightarrow \operatorname{Div}\left(\Gamma^{\prime}\right)$ 
by

$$
\varphi_{*}(D):=\sum_{x \in \Gamma} D(x) \cdot \varphi(x) .
$$

One can check that $\operatorname{deg}\left(\varphi_{*}(D)\right)=\operatorname{deg}(D)$ and $\varphi_{*}(\operatorname{div}(f))=\operatorname{div}\left(\varphi_{*} f\right)$ for any divisor $D$ on $\Gamma$ and any $f$ in $\operatorname{Rat}(\Gamma)$ (cf. [1, Proposition 4.2]).

\subsection{Chip-firing moves}

In [3], Haase, Musiker and Yu used the term subgraph of a tropical curve as a compact subset of the tropical curve with a finite number of connected components and defined the chip firing move $\mathrm{CF}\left(\widetilde{\Gamma_{1}}, l\right)$ by a subgraph $\widetilde{\Gamma_{1}}$ of a tropical curve $\widetilde{\Gamma}$ and a positive real number $l$ as the rational function $\operatorname{CF}\left(\widetilde{\Gamma_{1}}, l\right)(x):=-\min \left(l, \operatorname{dist}\left(x, \widetilde{\Gamma_{1}}\right)\right)$, where $\operatorname{dist}\left(x, \widetilde{\Gamma_{1}}\right)$ is the infimum of the lengths of the shortest path to arbitrary points on $\widetilde{\Gamma_{1}}$ from $x$. They proved that every rational function on a tropical curve is an (ordinary) sum of chip firing moves (plus a constant) ([3, Lemma 2]) with the concept of a weighted chip firing move. This is a rational function on a tropical curve having two disjoint proper subgraphs $\widetilde{\Gamma_{1}}$ and $\widetilde{\Gamma_{2}}$ such that the complement of the union of $\widetilde{\Gamma_{1}}$ and $\widetilde{\Gamma}_{2}$ in $\widetilde{\Gamma}$ consists only of open line segments and such that the rational function is constant on $\widetilde{\Gamma_{1}}$ and $\widetilde{\Gamma_{2}}$ and linear (smooth) with integer slopes on the complement. A weighted chip firing move is an (ordinary) sum of chip firing moves (plus a constant) ([3, Lemma 1]).

With unbounded edges, their definition of chip firing moves needs a little correction. Let $\Gamma_{1}$ be a subgraph of a tropical curve $\Gamma$ which does not have any connected components consisting only of points at infinity and $l$ a positive real number or infinity. The chip firing move by $\Gamma_{1}$ and $l$ is defined as the rational function $\mathrm{CF}\left(\Gamma_{1}, l\right)(x):=-\min \left(l, \operatorname{dist}\left(x, \Gamma_{1}\right)\right)$.

Lemma 2.4.1. A weighted chip firing move on a tropical curve is a linear combination of chip firing moves having integer coefficients (plus a constant).

Sketch of proof. We use the same notations as in their proof. All we have to do is to show the construction for the case with $l=\infty$. Especially, it is sufficient to check the case that $\Gamma_{1}$ consists only of points at infinity. Supposing that $\Gamma_{1}$ has only one point gives only two situations. Firstly, $\Gamma_{2}$ contains a finite point. Then $f$ can be written as $\pm s \cdot \operatorname{CF}\left(\Gamma_{2}, \infty\right)$ plus a constant, where $s$ is the slope of $f$ on the complement. Secondly, $\Gamma_{2}$ consists only of one point at infinity. Taking a finite point $x$, then $f$ can be written 
as $\pm s \cdot\left(\mathrm{CF}\left(f^{-1}([f(x), \infty]), \infty\right)-\mathrm{CF}(\{x\}, \infty)\right)$ plus a constant with same $s$ as the first situation. Suppose that $\Gamma_{1}$ has plural points. $\Gamma_{2}$ must contain at least one finite point. Let $x_{i}$ be the intersection of $\Gamma_{1}$ and the closure of $L_{i}$. Note that $\Gamma_{1}=\left\{x_{1}, \cdots, x_{k}\right\}$, where $k$ is no less than two. With the slope $s_{i}$ of $f$ on $e_{i}:=L_{i} \sqcup\left\{x_{i}\right\}, f$ is $\sum_{i=1}^{k}\left( \pm s_{i} \cdot \mathrm{CF}\left(\Gamma \backslash e_{i}, \infty\right)\right)$ plus a constant.

The next lemma is proven in the same way of [므, Lemma 2] and shows the appropriateness of this definition.

Lemma 2.4.2. Every rational function on a tropical curve is a linear combination of chip firing moves having integer coefficients (plus a constant).

A point on $\Gamma$ with valence two is said to be a smooth point. We sometimes refer to an effective divisor $D$ on $\Gamma$ as a chip configuration. We say that a subgraph $\Gamma_{1}$ of $\Gamma$ can fire on $D$ if for each boundary point of $\Gamma_{1}$ there are at least as many chips as the number of edges pointing out of $\Gamma_{1}$. A set of points on a tropical curve $\Gamma$ is said to be cut set of $\Gamma$ if the complement of that set in $\Gamma$ is disconnected.

\section{Generators of $R(D)^{K}$}

In this section, for an effective divisor $D$ on a tropical curve and a finite subgroup $K$ of the isometry group of the tropical curve, we find a generator set of the $K$-invariant set $R(D)^{K}$ of $R(D)$ and then, show that $R(D)^{K}$ is finitely generated as a tropical semimodule. When $D$ is $K$-invariant, $R(D) / \boldsymbol{R}$ is identified with the $K$-invariant linear system $|D|^{K}$, so $|D|^{K}$ is finitely generated by the generators of $R(D)^{K}$ modulo tropical scaling.

Remark 3.1 ([3, Lemma 6]). Let $\widetilde{\Gamma}$ be a tropical curve, $\widetilde{D}$ be a divisor on $\widetilde{\Gamma}$ and $S$ be the set of rational functions $f$ in $R(\widetilde{D})$ such that the support of $\widetilde{D}+\operatorname{div}(f)$ does not contain any cut set of $\widetilde{\Gamma}$ consisting only of smooth points. Then

(1) $S$ contains all the extremals of $R(\widetilde{D})$,

(2) $S$ is finite modulo tropical scaling, and

(3) $S$ generates $R(\widetilde{D})$ as a tropical semimodule. 
Though in the above remark they assume that $R(\widetilde{D})$ is a subset of $\boldsymbol{R}^{\widetilde{\Gamma}}$, the proof is applied even in the case that $R(\widetilde{D})$ is a subset of $(\boldsymbol{R} \cup\{ \pm \infty\})^{\widetilde{\Gamma}}$ with preparations in Section 2. Also, the above remark throws the relation between $S$ and $\widetilde{D}$ into relief, hence hereafter we write $S$ for $\widetilde{D}$ as $S(\widetilde{D})$. Note that a tropical subsemimodule of $R(\widetilde{D})$ is not always finitely generated. Consider the tropical subsemimodule of $R([0])$ corresponding to $|[0]| \backslash\{[0]\}$ on a tropical curve $[0,1]$.

Let $\Gamma$ be a tropical curve, $D$ an effective divisor on $\Gamma$ and $K$ a subgroup of the isometry group of $\Gamma$. One can expect the relation between $R(D)$ and $S(D)$ to be analogous to that of their $K$-invariant counterparts $R(D)^{K}$ and $S(D)^{K}$, but in vain. Indeed, the next example objects.

Example 3.2. Let $\widetilde{\Gamma}$ be a circle and let a map $i: \widetilde{G_{0}} \rightarrow \widetilde{G_{0}}$ which transfers two edges to each other, where $\widetilde{G_{0}}$ is the underlying graph of the canonical loopless model of $\widetilde{\Gamma}$. For a point $x_{1}$ on $\widetilde{\Gamma}$, we choose another point $x_{2}$ on $\widetilde{\Gamma}$ such that $i\left(x_{1}\right) \neq x_{2}$. For the group $\widetilde{K}$ generated by $i$ and the effective divisor $\widetilde{D}=x_{1}+x_{2}$, although $S(\widetilde{D})^{\widetilde{K}}$ is empty, $R(\widetilde{D})^{\widetilde{K}}$ is not empty. It means that $S(\widetilde{D})^{\widetilde{K}}$ is not a generator set of $R(\widetilde{D})^{\widetilde{K}}$.

Now, let us find a generator set for $R(D)^{K}$ that corresponds to $S(D)$ for $R(D)$. In the above situation, $K$ acts on $\Gamma$ naturally. We define $V_{1}(\Gamma)$ as the set of points $x$ on $\Gamma$ such that there exists a point $y$ in any neighborhood of $x$ whose stabilizer is not equal to that of $x$.

Lemma 3.3. $V_{1}(\Gamma)$ is a finite set.

Proof. We assume that $\Gamma$ is not the $\infty$-metric space obtained from only one edge with length of $\infty$. Let $\sigma: \Gamma \rightarrow \Gamma$ be an isometry. Then, for any edge $e$ of $\Gamma$, the image of $e$ by $\sigma$ agrees completely with $e$ or the intersection of $e$ and the image of $e$ by $\sigma$ is contained in the set of the endpoints of $e$. In fact, if $|e \cap \sigma(e)|$ is infinite, then $\sigma(e)$ is contained in $e$ because $e$ is an edge of $\Gamma$. It means that $\sigma(e)=e$. If $|e \cap \sigma(e)|$ is finite and $e \cap \sigma(e)$ contains a point on $\Gamma$ other than endpoints of $e$, then that point has the valence of greater than two. It contradicts to the fact that $e$ is an edge of $\Gamma$.

From the above argument, for any edge $e$ of $\Gamma$, we can roughly classify the situations into four. First, $\sigma$ is the identity map on $e$, i.e., $\sigma$ fixes all points on $e$. Second, $\sigma$ gives a mirror image of $e$. In this case, if $\Gamma$ is a circle consisting of $e$, the fixed points on $e$ by $\sigma$ are only antipodal points on the axis of symmetry of $\sigma$, otherwise, the midpoint of $e$ is fixed by $\sigma$, moreover 
when $e$ is a loop, then the vertex connected to $e$ is also fixed by $\sigma$. Third, $\sigma$ acts as a proper rotation on $e$. This is possible only when $\Gamma$ is a circle, and $\sigma$ gives no fixed points on $e$. Finally, $\sigma$ maps $e$ onto other edge of $\Gamma$, then only the endpoints of $e$ may be fixed by $\sigma$.

Consequently, under the above assumption, since $K$ is a finite set and $\Gamma$ has finite vertices and edges, $V_{1}(\Gamma)$ is a finite set.

Let us suppose that $\Gamma$ is the $\infty$-metric space obtained from only one edge with length of $\infty$. Since $K$ is a finite set, any $\sigma$ in $K$ is not a proper translation of $\Gamma$. Each isometry of $\Gamma$ other than translations fixes only one point on $\Gamma$. Thus, also in this case, $V_{1}(\Gamma)$ is a finite set. Note that there can exists only one inversion. If there were two distinct, these two can generate a translation, leading $|K|$ to infinity.

We set $\left(G_{0}, l_{0}\right)$ as the canonical loopless model of $\Gamma$. By Lemma 3.3, we obtain the model $\left(\widetilde{G_{1}}, \widetilde{l_{1}}\right)$ of $\Gamma$ by setting the $K$-orbit of the union of $V\left(G_{0}\right)$ and $V_{1}(\Gamma)$ as the set of vertices $V\left(\widetilde{G_{1}}\right)$. Naturally, we can regard that $K$ acts on $V\left(\widetilde{G_{1}}\right)$ and also on $E\left(\widetilde{G_{1}}\right)$. Thus, the sets $V\left(\widetilde{G^{\prime}}\right)$ and $E\left(\widetilde{G^{\prime}}\right)$ are defined as the quotient sets of $V\left(\widetilde{G_{1}}\right)$ and $E\left(\widetilde{G_{1}}\right)$ by $K$, respectively. Let $\widetilde{G^{\prime}}$ be the graph obtained by setting $V\left(\widetilde{G^{\prime}}\right)$ as the set of vertices and $E\left(\widetilde{G^{\prime}}\right)$ as the set of edges. Since $\widetilde{G_{1}}$ is connected, $\widetilde{G^{\prime}}$ is also connected. We obtain the loopless graph $G^{\prime}$ from $\widetilde{G^{\prime}}$ and the loopless model $\left(G_{1}, l_{1}\right)$ of $\Gamma$ from the inverse image of $V\left(G^{\prime}\right)$ by the map defined by $K$. Note that $V\left(G_{1}\right)$ contains $V\left(\widetilde{G_{1}}\right)$. Since $K$ is a finite subgroup of the isometry group of $\Gamma$, the length function $l^{\prime}: E\left(G^{\prime}\right) \rightarrow \boldsymbol{R}_{>0} \cup\{\infty\},[e] \mapsto\left|K_{e}\right| \cdot l_{1}(e)$ is well-defined, where [e] and $K_{e}$ mean the equivalence class of $e$ and the stabilizer of $e$, respectively. Let $\Gamma^{\prime}$ be the tropical curve obtained from $\left(G^{\prime}, l^{\prime}\right)$. Then, $\Gamma^{\prime}$ is the quotient tropical curve of $\Gamma$ by $K$.

For any edge $e$ of $G_{1}$, by the Orbit-Stabilizer formula, $\left|K_{e}\right|$ is a positive integer. Thus, for $\left(G_{1}, l_{1}\right)$ and $\left(G^{\prime}, l^{\prime}\right)$, there exists only one morphism $\varphi$ : $\Gamma \rightarrow \Gamma^{\prime}$ that satisfies $\operatorname{deg}_{e}(\varphi)=\left|K_{e}\right|$ for any edge $e$ of $G_{1}$.

We obtain the following lemma as an extension of [2, Lemma 2.2].

Lemma 3.4. If both $\Gamma$ and $\Gamma^{\prime}$ are not singletons, then $\varphi$ is a finite harmonic morphism of degree $|K|$.

Proof. Clearly, $\varphi$ is finite. Now we check that $\varphi$ is harmonic and its degree is $|K|$. Since $K$ is a finite subgroup of the isometry group of $\Gamma$, for any point $x$ on $\Gamma$ and any half-edge $h^{\prime}$ of $\varphi(x)$, each connected component of $\varphi^{-1}\left(h^{\prime}\right)$ 
has the same dilation factor $\operatorname{deg}_{h}(\varphi)$, where $h$ is a connected component emanating from $x$. Therefore, for the edge $e$ of $G_{1}$ containing $h$ and its image $e^{\prime}$ by $\varphi$, the following hold:

$$
\operatorname{deg}_{x}(\varphi)=\sum_{\widetilde{h} \mapsto h^{\prime}} \operatorname{deg}_{h}(\varphi)=\sum_{\widetilde{e} \mapsto e^{\prime}} \operatorname{deg}_{e}(\varphi)=|K e| \cdot\left|K_{e}\right|=|K| .
$$

Where $\widetilde{h}, \widetilde{e}$ and $K e$ denote a connected component of $\varphi^{-1}\left(h^{\prime}\right)$, that of $\varphi^{-1}\left(e^{\prime}\right)$ and the orbit of $e$ by $K$, respectively. Note that we use the Orbit-Stabilizer formula at the last equality. Accordingly, we get the conclusion.

Note that whether $\Gamma$ is a singleton or not agrees with whether $\Gamma^{\prime}$ is a singleton.

Is $R(D)^{K}$, the $K$-invariant set of $R(D)$, identical to $\varphi^{*}\left(R\left(\varphi_{*}(D)\right)\right)$ ? Nor is it.

Example 3.5. Assume the situation of Example 3.2. For a rational function $f$ which decreases from $\varphi\left(x_{1}\right)$ to $\varphi\left(x_{2}\right)$ with slope one and is constant on other graph, however $f$ is an element of $R\left(\varphi_{*}(\widetilde{D})\right)$, the pull-back of $f$ by $\varphi$ is not in $R(\widetilde{D})^{\widetilde{K}}$.

Next, for $R(D)^{K}$, the following holds.

Lemma 3.6. $R(D)^{K}$ is a tropical semimodule.

Proof. Let $c$ be in $\boldsymbol{R}, f, g$ in $R(D)^{K}$ and $\sigma$ in $K$. Since $R(D)$ is a tropical semimodule by Lemma 2.2.1, $c \odot f$ and $f \oplus g$ are in $R(D)$. It is obvious that $\odot$ and $\circ$ are associative and that $\circ$ is distributive over $\oplus$ from right, both $(c \odot f) \circ \sigma$ and $(f \oplus g) \circ \sigma$ are in $R(D)^{K}$.

Note that $R(D+\operatorname{div}(f))^{K}=R(D)^{K} \odot(-f)$ for any $K$-invariant rational function $f$.

The following lemma is an extension of [3], Lemma 5].

Lemma 3.7. Let $f$ be in $\operatorname{Rat}(\Gamma)$. Then, $f$ is an extremal of $R(D)^{K}$ if and only if there are not two proper $K$-invariant subgraphs $\Gamma_{1}$ and $\Gamma_{2}$ covering $\Gamma$ such that each can fire on $D+\operatorname{div}(f)$.

Proof. First, let us show the "if" part. Suppose that there are two such subgraphs $\Gamma_{1}$ and $\Gamma_{2}$. We can assume that each $\Gamma_{i}$ does not have any connected component consisting only of points at infinity. Each $\Gamma_{i}$ defines a chip firing 
move $g_{i}$ for a small positive number so that $g_{i}$ is zero on $\Gamma_{i}$ and they are nonpositive. As $\Gamma_{1}$ and $\Gamma_{2}$ are $K$-invariant, so $g_{1}$ and $g_{2}$ are in $R(D+\operatorname{div}(f))^{K}$. Since $g_{1} \oplus g_{2}=0$ on $\Gamma$, we can write $f$ as $\left(f+g_{1}\right) \oplus\left(f+g_{2}\right)$, i.e. $f$ is not an extremal of $R(D)^{K}$.

Next, let us show the "only if" part. Suppose $f=g_{1} \oplus g_{2}$ for some $g_{1}$ and $g_{2}$ in $R(D)^{K} \backslash\{f\}$. For $i=1,2$, there exists $\widetilde{g}_{i}$ in $R(D+\operatorname{div}(f))^{K}$ such that $g_{i}=\widetilde{g}_{i} \odot f$. Let $\Gamma_{i}$ be the closure of the loci where $\widetilde{g}_{i}=0$. Then, the union of $\Gamma_{1}$ and $\Gamma_{2}$ is $\Gamma$ and each $\Gamma_{i}$ is proper. Since $\widetilde{g}_{i}$ is $K$-invariant, so is $\Gamma_{i}$. Then, each $\Gamma_{i}$ can fire on $D+\operatorname{div}(f)$.

The term "a subgraph is infinite" means that the subgraph is a infinite set.

Lemma 3.8. Let $A$ be a $K$-invariant subset of $\operatorname{supp}(D)$. If $\varphi(A)$ is a cut set of $\Gamma^{\prime}$ and $D(x) \geq \operatorname{val}(x)-1$ for any $x$ in $A$, then there exists a $K$-invariant infinite subgraph $\Gamma_{1}$ of $\Gamma$ which can fire on $D$ and whose boundary points are in $A$.

Proof. For such $A$, let $\Gamma_{1}^{\prime}, \cdots, \Gamma_{n}^{\prime}$ be distinct connected components of $\Gamma^{\prime} \backslash$ $\varphi(A)$ respectively. Note that $n$ is no less than two since $\varphi(A)$ is a cut set of $\Gamma^{\prime}$. Clearly, for any $i$, the inverse image of the closure of $\Gamma_{i}^{\prime}$ by $\varphi$ is a $K$-invariant infinite subgraph of $\Gamma$ we want.

We call a point on $\Gamma$ not being a vertex of $G_{1}$ a $K$-ordinary point. Note that if a subgraph of $\Gamma$ has a $K$-ordinary point, topologically saying, it should have infinite points.

Lemma 3.9. Let $\Gamma_{1}$ be a $K$-invariant subgraph of $\Gamma$. If $\Gamma_{1}$ is infinite and if the set of its boundary points $\partial \Gamma_{1}$ contains at least one $K$-ordinary point, then $\varphi\left(\partial \Gamma_{1}\right)$ is a cut set of $\Gamma^{\prime}$ and contains a point on $\Gamma^{\prime}$ not being a vertex of $G^{\prime}$.

Proof. For such $\Gamma_{1}$, obviously $\varphi\left(\partial \Gamma_{1}\right)$ contains a point on $\Gamma^{\prime}$ not being a vertex of $G^{\prime}$. It is sufficient to check that $\varphi\left(\partial \Gamma_{1}\right)$ is a cut set of $\Gamma^{\prime}$. Let $\Gamma_{2}$ be the closure of the complement set of $\Gamma_{1}$ in $\Gamma$. This $\Gamma_{2}$ is $K$-invariant and contains a $K$-ordinary point. Thus, $\Gamma_{2}$ is an infinite subgraph. Consequently, $\Gamma^{\prime} \backslash \varphi\left(\partial \Gamma_{1}\right)=\varphi\left(\Gamma_{1} \cup \Gamma_{2}\right) \backslash \varphi\left(\partial \Gamma_{1}\right)=\left(\varphi\left(\Gamma_{1}\right) \backslash \varphi\left(\partial \Gamma_{1}\right)\right) \sqcup\left(\varphi\left(\Gamma_{2}\right) \backslash \varphi\left(\partial \Gamma_{1}\right)\right)$. Hence, $\varphi\left(\partial \Gamma_{1}\right)$ is a cut set of $\Gamma^{\prime}$.

The next corollary follows from Lemma 3.8 and Lemma 3.9. 
Corollary 3.10. For a subset of the support of $\varphi_{*}(D)$, we consider the following condition $(*)$ :

(*) it is a cut set of $\Gamma^{\prime}$ containing no vertices of $G^{\prime}$ and whose inverse image by $\varphi$ is a subset of the support of $D$.

(1) For a subset $A$ of $\operatorname{supp}(D)$ whose image by $\varphi$ satisfies $(*)$, there exists a $K$-invariant infinite subgraph $\Gamma_{1}$ of $\Gamma$ which can fire on $D$ and whose boundary points are in $A$.

(2) Let $\Gamma_{1}$ be a $K$-invariant subgraph of $\Gamma$. If $\Gamma_{1}$ is infinite and can fire on $D$ and if the set of its boundary points consists only of $K$-ordinary points, then the image of the set of boundary points of $\Gamma_{1}$ by $\varphi$ satisfies $(*)$.

By Corollary 3.10 , it is natural to define $S(D)_{K}$ as the set of $f$ in $R(D)^{K}$ such that there exist no cut sets of $\Gamma^{\prime}$ contained in the support of $\varphi_{*}(D+$ $\operatorname{div}(f))$, containing no vertices of $G^{\prime}$ and whose inverse image by $\varphi$ is a subset of the support of $D+\operatorname{div}(f)$. In fact, this $S(D)_{K}$ is the set corresponding to $S(D)$, i.e. $S(D)_{K}$ is a generator set of $R(D)^{K}$.

Theorem 3.11. In the above situation, the following hold:

(1) $S(D)_{K}$ contains all the extremals of $R(D)^{K}$,

(2) $S(D)_{K}$ is finite modulo tropical scaling, and

(3) $S(D)_{K}$ generates $R(D)^{K}$ as a tropical semimodule.

Proof. (1) Suppose $f$ is in the difference set of $R(D)^{K}$ from $S(D)_{K}$, then there exists a cut set $A^{\prime}$ of $\Gamma^{\prime}$ contained in $\operatorname{supp}\left(\varphi_{*}(D+\operatorname{div}(f))\right)$, containing no vertices of $G^{\prime}$ and such that $\varphi^{-1}\left(A^{\prime}\right) \subset \operatorname{supp}(D+\operatorname{div}(f))$. By (1) of Corollary 3.10 , there exists a $K$-invariant infinite subgraph $\Gamma_{1}$ of $\Gamma$ which can fire on $D+\operatorname{div}(f)$ and whose boundary points are in $\varphi^{-1}\left(A^{\prime}\right)$. Then, the closure of $\Gamma \backslash \Gamma_{1}$ can also fire on $D+\operatorname{div}(f)$. Therefore, by Lemma 3.7, $f$ is not an extremal of $R(D)^{K}$.

(2) The push-forward of a rational function on $\Gamma$ induces a natural map $S(D)_{K} / \boldsymbol{R} \rightarrow S\left(\varphi_{*}(D)\right) / \boldsymbol{R},[f] \mapsto\left[\varphi_{*}(f)\right]$. In fact, for any $f$ in $S(D)_{K}$, $\varphi_{*}(D+\operatorname{div}(f))=\varphi_{*}(D)+\varphi_{*}(\operatorname{div}(f))=\varphi_{*}(D)+\operatorname{div}\left(\varphi_{*}(f)\right)$, thus, $\varphi_{*}(f)$ is in $R\left(\varphi_{*}(D)\right)$. From $f \in S(D)_{K}$, there exist no cut sets of $\Gamma^{\prime}$ contained 
in $\operatorname{supp}\left(\varphi_{*}(D)+\varphi_{*}(\operatorname{div}(f))\right)$, containing no vertices of $G^{\prime}$ and whose inverse image by $\varphi$ is a subset of $\operatorname{supp}(D+\operatorname{div}(f))$. This means that $\varphi_{*}(f)$ is in $S\left(\varphi_{*}(D)\right)$. Also, for any pair of $f_{1}$ and $f_{2}$ in $[f]$, there exists $c$ in $\boldsymbol{R}$ satisfying $f_{2}=f_{1}+c$. Since $\varphi_{*}\left(f_{2}\right)=\varphi_{*}\left(f_{1}+c\right)=\varphi_{*}\left(f_{1}\right)+\varphi_{*}(c)=\varphi_{*}\left(f_{1}\right)+c$, the map is well-defined. Now we show that the map is injective. Let $[f]$ and $[g]$ be distinct elements of $S(D)_{K} / \boldsymbol{R}$, thus $\operatorname{div}(f) \operatorname{differs}$ from $\operatorname{div}(g)$. Since both $f$ and $g$ are $K$-invariant, so their images $\varphi_{*}(\operatorname{div}(f))$ and $\varphi_{*}(\operatorname{div}(g))$ are different, i.e. the map is injective. By Remark 3.1, we get the conclusion.

(3) Suppose $f \in R(D)^{K}$. Let $N(f)$ be the number of distinct $K$-orbits in the union of all $K$-invariant subsets of $\operatorname{supp}(D+\operatorname{div}(f))$ which is a cut set of $\Gamma^{\prime}$ containing no vertices of $G^{\prime}$. We prove (3) by induction for $N(f)$. If $N(f)=0$, then $f \in S(D)_{K}$ from the definition of $S(D)_{K}$. Assume that $f \in$ $\left\langle S(D)_{K}\right\rangle$ for all $N(f) \leq k$, where $\left\langle S(D)_{K}\right\rangle$ means the tropical semimodule generated by $S(D)_{K}$. We consider the case where $N(f)=k+1$ and $f \notin$ $S(D)_{K}$. Let $A$ be a subset of $\operatorname{supp}(D+\operatorname{div}(f))$ whose image by $\varphi$ is a cut set of $\Gamma^{\prime}$ containing no vertices of $G^{\prime}$. By (1) of Corollary [3.10, there exists a $K$-invariant subgraph $\Gamma_{1}$ of $\Gamma$ which can fire on $D+\operatorname{div}(f)$ and whose boundary points are in $A$. Let $\Gamma_{2}$ be the closure of the complement of $\Gamma_{1}$ in $\Gamma$. For any $x \in \partial \Gamma_{i}$, we write the distance between $x$ and its closest vertex of $G_{1}$ as $l_{x_{i}}$. Let $l_{i}:=\min \left\{l_{x_{i}} \mid x \in \partial \Gamma_{i}\right\}$ and $g_{i}:=\operatorname{CF}\left(\Gamma_{i}, l_{i}\right)$. Then, for both $i=1,2, f \odot g_{i}$ is not equal to $f$ and is in $R(D)^{K}$ since $f, g_{i} \in R(D)^{K}$ and $f=\left(f \odot g_{1}\right) \oplus\left(f \odot g_{2}\right)$. By the definition of $g_{i}, N(f)>N\left(f \odot g_{i}\right)$ and $f \odot g_{i} \in\left\langle S(D)_{K}\right\rangle$, then $f \in\left\langle S(D)_{K}\right\rangle$.

By Lemma 2.2.3 and the above theorem, we obtain the following corollary, which is an extension of [3, Corollary 9].

Corollary 3.12. Let $\Gamma$ be a tropical curve, $D$ an effective divisor on $\Gamma$ and $K$ a finite subgroup of the isometry group of $\Gamma$. Then, the tropical semimodule $R(D)^{K}$ is generated by the extremals of $R(D)^{K}$. This generating set is minimal and unique up to tropical scalar multiplication.

If $D$ is $K$-invariant, $R(D)^{K} / \boldsymbol{R}$ is naturally identified with the $K$-invariant linear subsystem $|D|^{K}$. In conclusion, the following statement holds from Theorem 3.11,

Theorem 3.13. Let $\Gamma$ be a tropical curve, $D$ an effective divisor on $\Gamma$ and $K$ a finite subgroup of the isometry group of $\Gamma$. If $D$ is $K$-invariant, then the $K$-invariant linear subsystem $|D|^{K}$ of $|D|$ is finitely generated by $S(D)_{K} / \boldsymbol{R}$. 


\section{References}

[1] Mathew Baker, Serguei NorineHarmonic Morphisms and Hyperelliptic Graphs, Int. Math. Res. Not. 15 (2009)2914-2955.

[2] Melody Chan, Tropical curves and metric graphs, Univ. of California, Berkeley thesis, 2012.

[3] Christian Haase, Gregg Musiker, Josephine YuLinear Systems on Tropical Curves, Mathematische Zeitschrift 270 (2012), 1111-1140.

[4] Yuki Kageyama, Divisorial condition for the stable gonality of tropical curves, arXiv:1801.07405.

[5] Shu Kawaguchi, Kazuhiko Yamaki, Rank of Divisors Under SpecializationInt. Math. Res. Not. 12 (2015)4121-4176.

[6] Mizuho Shinjo, gonality of nonsingular tropical curves with genus three, master's thesis of Tokyo Metropolitan University, 2017

(https://tokyo-metro-u.repo.nii.ac.jp/index.php?action=repository_view _main_item_detail\&item_id=6422\&item_no=1\&page_id=30\&block_id=15 $5)$. 